\title{
Review Article \\ Effects of ACEIs and ARBs on the Residual Renal Function in Peritoneal Dialysis Patients: A Meta-Analysis of Randomized Controlled Trials
}

\author{
Lei Ding, Jingjuan Yang, Lizhu Li, and Yi Yang \\ Department of Nephrology, The Fourth Affiliated Hospital, College of Medicine, Zhejiang University, N1 Shangcheng Road, Yiwu, \\ Zhejiang, China \\ Correspondence should be addressed to Yi Yang; yangyixk@zju.edu.cn
}

Received 27 April 2020; Accepted 21 August 2020; Published 24 September 2020

Academic Editor: Stavros Baloyannis

Copyright ( 2020 Lei Ding et al. This is an open access article distributed under the Creative Commons Attribution License, which permits unrestricted use, distribution, and reproduction in any medium, provided the original work is properly cited.

\begin{abstract}
Background. In peritoneal dialysis (PD) patients, whether angiotensin-converting enzyme inhibitors (ACEIs) and angiotensin receptor blockers (ARBs) could protect residual renal function is still controversial. To assess the effects of ACEIs and ARBs on the residual renal function and cardiovascular (CV) events in peritoneal dialysis patients, we performed a meta-analysis of randomized controlled trials. Materials and Methods. We searched PubMed, EMBASE, the Cochrane Library, the CNKI database, and the Wanfang database for relevant articles from database inception to November 30, 2019. Randomized controlled trials were included. The primary outcome was the decline in the residual renal function (RRF). Results. Thirteen trials with 625 participants were included in the meta-analysis. The average residual GFR declined by $1.79 \mathrm{ml} / \mathrm{min}$ per $1.73 \mathrm{~m}^{2}$ in the ACEI/ARB group versus $1.44 \mathrm{ml} / \mathrm{min}$ per $1.73 \mathrm{~m}^{2}$ in the placebo or active control group at $3 \mathrm{mo}$. The average residual GFR declined by 2.02 versus $2.06,2.16$ versus 2.72 , and -0.04 versus $2.74 \mathrm{ml} / \mathrm{min}$ per $1.73 \mathrm{~m}^{2}$ in the placebo or active control group at 6 months (mo), $12 \mathrm{mo}$, and $24 \mathrm{mo}$, respectively. The decline in residual GFR showed a significant difference between the ACEI/ARB group and the placebo or active control group at $12 \mathrm{mo}\left(\mathrm{MD}=-0.64 \mathrm{ml} / \mathrm{min}\right.$ per $1.73 \mathrm{~m}^{2} ; 95 \% \mathrm{CI}:-0.97 \sim-0.32 ; I^{2}=$ $44 \% ; P<0.0001)$. No significant difference was observed in $\mathrm{Kt} / \mathrm{V}$, urinary protein excretion, weekly creatinine clearance, $\mathrm{CV}$ events, or serum potassium levels. Conclusions. In the present study, we found that the use of ACEIs and ARBs, especially longterm treatment, decreased the decline of RRF in patients on PD. ACEIs and ARBs do not cause an additional risk of side effects.
\end{abstract}

\section{Introduction}

Cardiovascular (CV) disease is the main leading cause of death in patients with end-stage renal disease (ESRD), which accounts for over $40 \%$ deaths in dialysis patients [1]. Previous studies demonstrated that using angiotensinconverting enzyme inhibitors (ACEIs) and angiotensin receptor blockers (ARBs) could reduce the morbidity of cardiovascular events and mortality in chronic kidney disease (CKD) patients $[2,3]$.

In peritoneal dialysis (PD), the preserved residual renal function (RRF) is significantly associated with the better CV outcomes and lower mortality [4-6], mainly due to better control of malnutrition and hypertension, less ventricular hypertrophy, and lower rates of infection and hospitalization $[7,8]$. These date suggest that preserve RRF in PD patients may be critical. Previous studies found that ACEIs and ARBs might preserve the residual renal function via decreasing inflammation and glomerulosclerosis in PD patients [9-11]. However, most studies aimed at evaluating the effects of ACEI and ARB therapy in dialysis patients provided different consequences and much uncertainty about the protective effects of these medications persists $[12,13]$. Therefore, there is still no reliable evidence to confirm whether ACEIs/ARBs are worthy of clinical promotion in PD patients.

In the present study, randomized controlled studies (RCTs) using ACEIs/ARBs in PD patients were systematically evaluated in this meta-analysis to evaluate whether 
ACEIs and ARBs could protect the residual renal function in PD patients and whether they could provide evidence and promote application in PD patients.

\section{Material and Methods}

2.1. Date Sources, Search Strategy, and Selection Criteria. We searched PubMed, EMBASE, the Cochrane Library, the CNKI database, and the Wanfang database for relevant articles from database inception to November 30, 2019. We used the MeSH headings and text words of all spellings of known ACEIs and ARBs and peritoneal dialysis (Additional file 1). Randomized controlled trials without language limitations were included.

Eligible studies had the following characteristics: (1) studies of human subjects with RCTs, (2) the subjects in the study were composed of peritoneal dialysis patients over 18 years of age, (3) studies with documented data on renal outcomes or cardiovascular events, and (4) studies with a treatment duration of more than 3 months (mo).

2.2. Data Extraction and Quality Assessment. Published reports were obtained for each eligible trial, and relevant information extracted into a spreadsheet. The following data were extracted: first author, study characteristics (publication year, country, duration, setting, and design), and participant characteristics (interventions, age, sex, and sample size). The primary outcome was the decline in RRF. The secondary outcomes were anuria, change of $\mathrm{Kt} / \mathrm{V}$, urinary protein extraction, weekly creatine clearance, and cardiovascular events. RRF was measured by the glomerular filtration rate (GFR) or 24-hour urinary urea and creatinine clearances. Anuria was defined as total absence of urine output. Cardiovascular events included death from cardiovascular causes, nonfatal myocardial infarction, cerebrovascular events with permanent neurologic deficit, and peripheral vascular disease requiring lower-limb amputation above the ankle.

The literature were searched and identified by two investigators (LD and JJY) independently. Data extraction and quality assessment were undertaken independently by two investigators (LD and LZL) using a standardized approach. Any disagreement between the two investigators in the abstracted data was adjudicated by a third reviewer (YY). For studies with insufficient information, the reviewers contacted the primary authors, when possible, to acquire and verify the data.

2.3. Risk of Bias Assessment. The methodological quality of RCTs was assessed using the Cochrane risk of bias tool.

2.4. Statistical Analysis. For each eligible study, dichotomous data were analyzed by using the risk ratio (RR), which was computed using the Mantel-Haenszel method (fixed- or random-effects models). Continuous outcomes measured on the same scale are expressed as a mean value and standard deviation and were analyzed by using weighted mean differences (WMDs). The $I$-squared $\left(I^{2}\right)$ test was performed to assess the impact of study heterogeneity on the results of the meta-analysis. According to the Cochrane review guidelines, if severe heterogeneity was present, indicated by $I^{2}>$
$50 \%$, then the random-effect models were chosen. Otherwise, the fixed-effect models were used. A sensitivity analysis was performed if low-quality trials were identified. The overall effect was tested using $Z$ scores calculated by Fisher's $Z$ transformation, with significance set at $P<0.05$. Publication bias was assessed with funnel plots. Data analyses were performed by using Review Manager 5.3 (Cochrane Collaboration, Oxford, UK).

\section{Results}

After searching the electronic databases and selecting the relevant citations, 5630 studies were identified. Browsing the headlines and summaries, we performed full manuscript reviews of the remaining 117 articles. Thirteen reports were included in the meta-analysis (Figure 1).

3.1. Baseline Characteristics of the Patients Included. Table 1 describes the characteristics of the 13 included studies with 625 participants in total $[9,10,14-24]$. These studies were performed between 2003 to 2016. Three studies $(n=165)$ compared ACEIs with active controls $[9,18,23]$, one study $(n=90)$ compared ACEIs with ARBs [19], eight studies $(n=346)$ compared ARBs with active controls [10, 14-17, $20,22,24]$, and one studies $(n=24)$ compared ARBs with a placebo [21].

3.2. Risk of Bias. The quality of the included studies was estimated using the Cochrane Collaboration tool for assessing the risk of bias; a low versus high risk of bias is indicated for each study in Figure 2.

3.3. Decline of the Residual Renal Function. Data regarding the effects of ACEIs/ARBs on RRF were available in 10 trials $[9,10,14,16-19,22-24]$. The average residual GFR declined by $1.79 \mathrm{ml} / \mathrm{min}$ per $1.73 \mathrm{~m}^{2}$ in the ACEI/ARB group versus $1.44 \mathrm{ml} / \mathrm{min}$ per $1.73 \mathrm{~m}^{2}$ in the placebo or active control group at 3 mo. The average residual GFR declined by 2.02 versus $2.06,2.16$ versus 2.72 , and -0.04 versus $2.74 \mathrm{ml} / \mathrm{min}$ per $1.73 \mathrm{~m}^{2}$ in the placebo or active control group at $6 \mathrm{mo}$, $12 \mathrm{mo}$, and $24 \mathrm{mo}$, respectively. The decline in residual GFR showed a significant difference between the ACEI/ARB group and the placebo or active control group at $12 \mathrm{mo}$ $\left(\mathrm{MD}=-0.64 \mathrm{ml} / \mathrm{min}\right.$ per $1.73 \mathrm{~m}^{2} ; 95 \% \mathrm{CI}$ : $-0.97 \sim-0.32 ; I^{2}$ $=44 \% ; P<0.0001)$ (Figure 3 and Additional Figure 1).

3.4. Anuria. There were 6 studies described the change in urine volume between the ACEI/ARB group and the control group [16-18, 22-24]. Three studies reported the anuria [9, $22,24]$. The decline in urine volume showed a significant difference between the ACEI/ARB group and the active control group $\left(\mathrm{MD}=-224.94 \mathrm{ml} / \mathrm{d}\right.$; 95\% CI: $-343.94 \sim-105.94 ; I^{2}=$ $77 \% ; P<=0.0002$ ) (Figure 4). However, there was no significant difference in the effect of anuria (Additional Figure 2).

3.5. Change of $K t / V$. The average change in $\mathrm{Kt} / \mathrm{V}$ declined by 0.07 in the ACEI/ARB group versus 0.17 in the placebo or active control group (Additional Figure 3). 


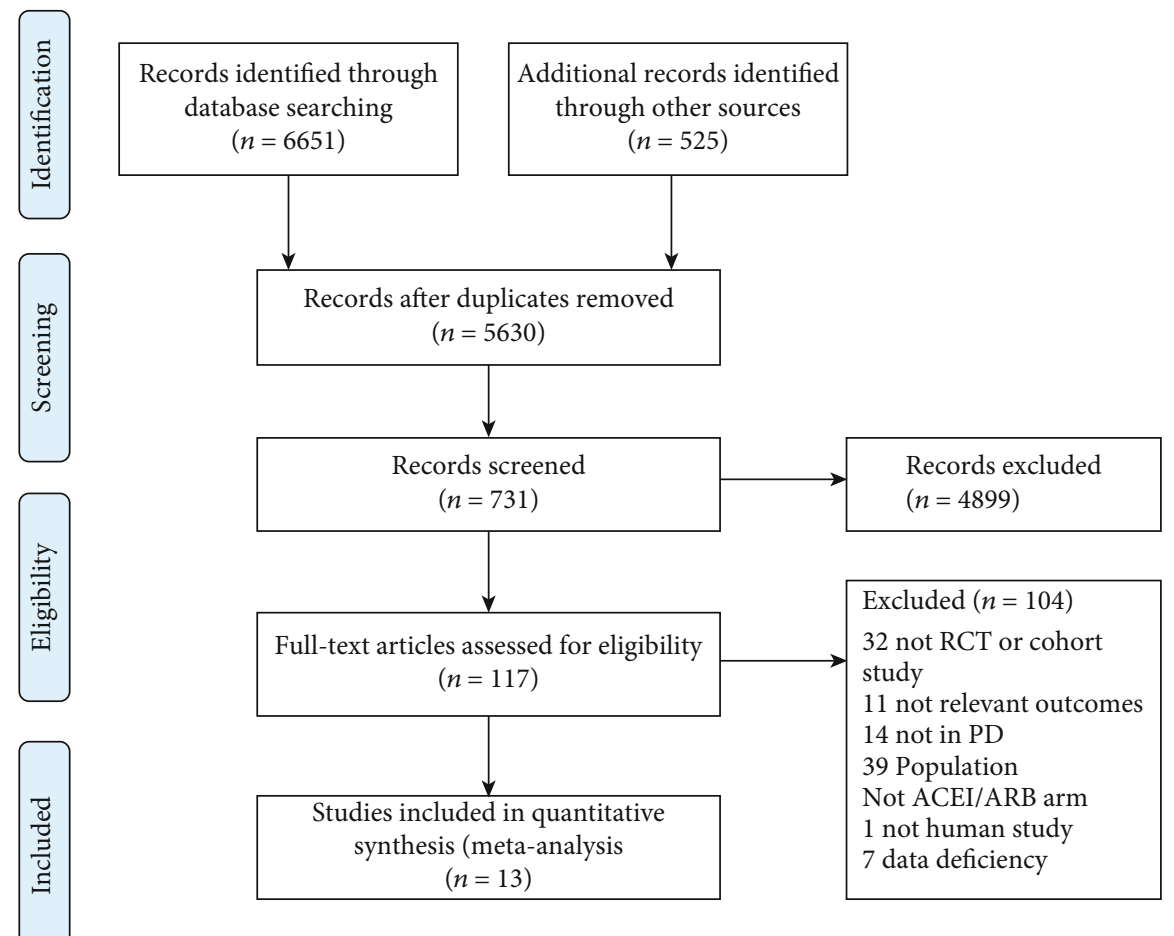

Figure 1: PRISMA 2009 flow diagram.

3.6. Change in Urinary Protein Excretion. The average change in urinary protein excretion declined by $-0.10 \mathrm{~g} / \mathrm{d}$ in the $\mathrm{ACEI} / \mathrm{ARB}$ group versus $0.65 \mathrm{~g} / \mathrm{d}$ in the placebo or active control group (Additional Figure 4).

3.7. Change of Weekly Creatinine Clearance. The average change in weekly creatinine clearance declined by $2.86 \mathrm{~L} / \mathrm{wk}$ per $1.73 \mathrm{~m}^{2}$ in the ACEI/ARB group versus $7.76 \mathrm{~L} /$ wk per $1.73 \mathrm{~m}^{2}$ in the placebo or active control group (Additional Figure 5).

\section{Discussion}

In this quantitative systematic review comprising of 13 trials and 625 participants, we found that both ACEIs and ARBs showed a significant benefit in preserving RRF in PD patients at 12 months. There was no significant benefit when shortterm ACEIs/ARBs were used ( $\leq 6$ months). No significant difference was observed in $\mathrm{Kt} / \mathrm{V}$, urinary protein excretion, weekly creatinine clearance, or serum potassium levels.

It has been reported that maintenance of RRF is independently correlated with increased survival in ESRD patients [25]. Preservation of RRF is also associated with improved volume and nutritional status, better blood pressure control, reduced erythropoietin requirements, and lower risk of inflammation [26]. Therefore, preservation of RRF might be a potential therapeutic target in patients on $\mathrm{PD}$.

In PD patients, whether ACEIs and ARBs could protect residual renal function is still controversial. In a RCT including 60 incident PD patients by Li et al., the use of ACEI could slow the decline in RRF [9]. Similarly, Suzuki et al. also suggested that patients undergoing PD with ARB preserved RRF, compared with the control groups [10]. In contrast to these beneficial effects of ACEIs or ARBs on the preserved RRF, a recent retrospective observational analysis in the United States demonstrated that ACEI/ARB use might not reduce the decline of RRF in PD patients [27]. Another observational cohort study of patients initiating PD in the Netherlands also found there were no renoprotective effects of ACEIs/ARBs in PD patients [28]. There were many risk factors affecting the RRF in patients on PD, including PD prescription [29], PD solution types [30], volume status [31], use of diuretics [32], and nephrotoxic agents [33]. Many dialysis patients have more than one of these risk factors so that these confounding factors might modify the beneficial effect of ACEIs/ARBs on preservation of RRF in observational studies.

In the present study, we found the long-term use (12 months) of ACEIs/ARBs presented benefit in preserving RRF in PD patients, although there was no significant benefit when short-term ACEIs/ARBs were used ( $\leq 6$ months). Dialysis patients experience abnormal response of the reninangiotensin system (RAS), leading to higher incidence of hypertension, which is one of the leading causes of $\mathrm{CV}$ disease and mortality [34]. In an analysis of over 110,000 dialysis patients, the benefit of ACEI/ARB usage was greater in patients who use agents for longer duration [35]. Longterm usage of RAS blockade might help to maintain stable blood pressure and inhibit the RAS system, thereby protecting the residual renal function in dialysis patients.

Our systematic review had some strengths. We analyzed the changes in the residual renal function at different time points and compared not only renal function progression but cardiovascular events in PD patients. Compared with 


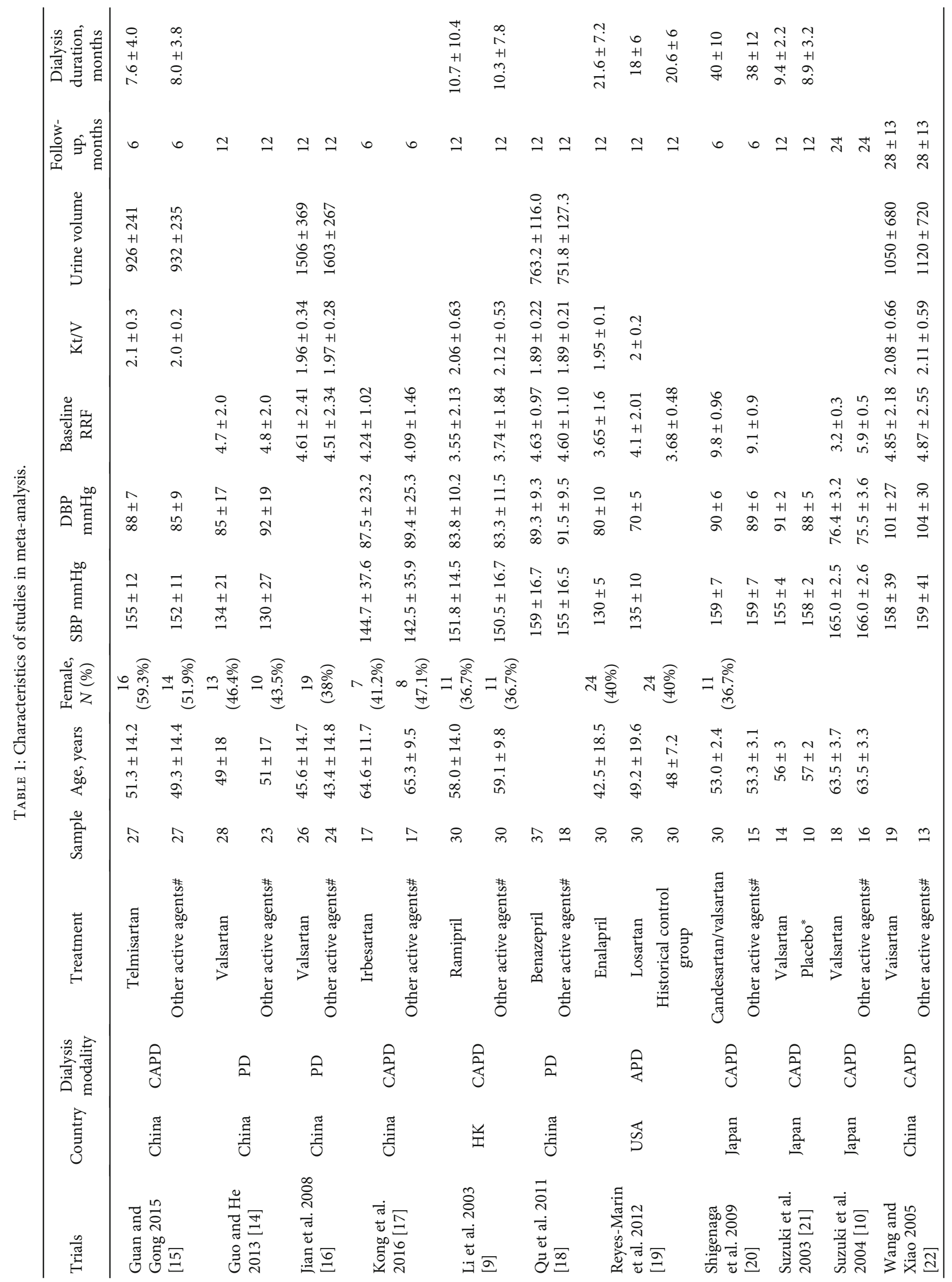




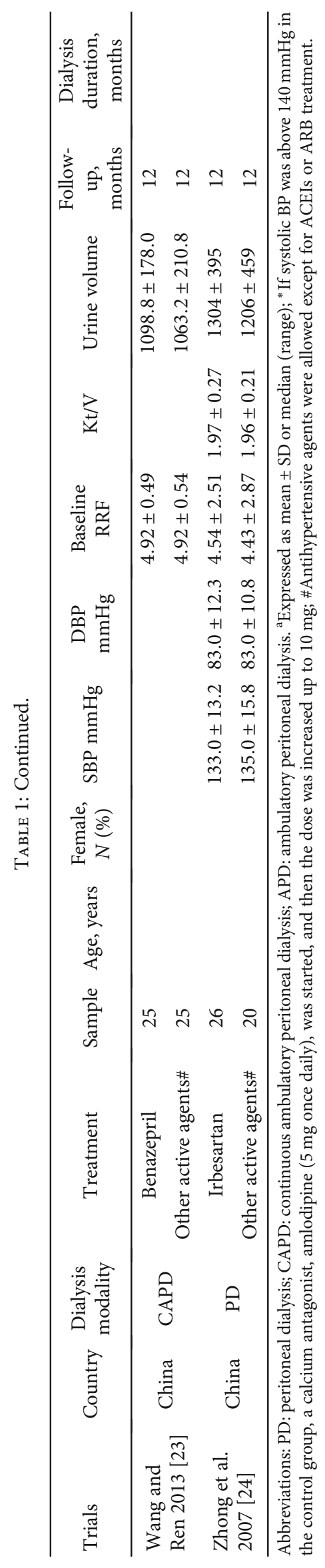




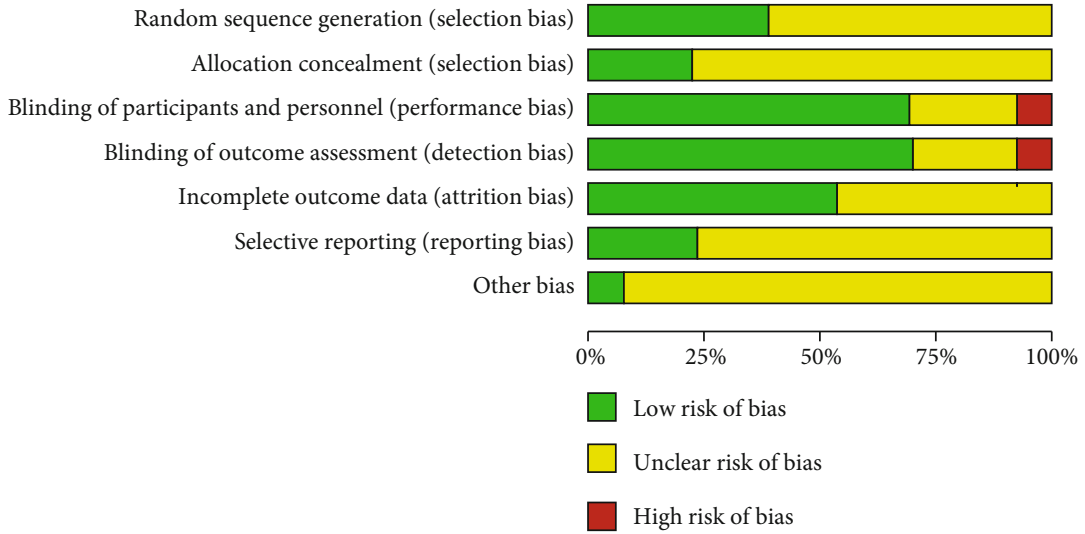

Figure 2: Quality assessment for included trials.

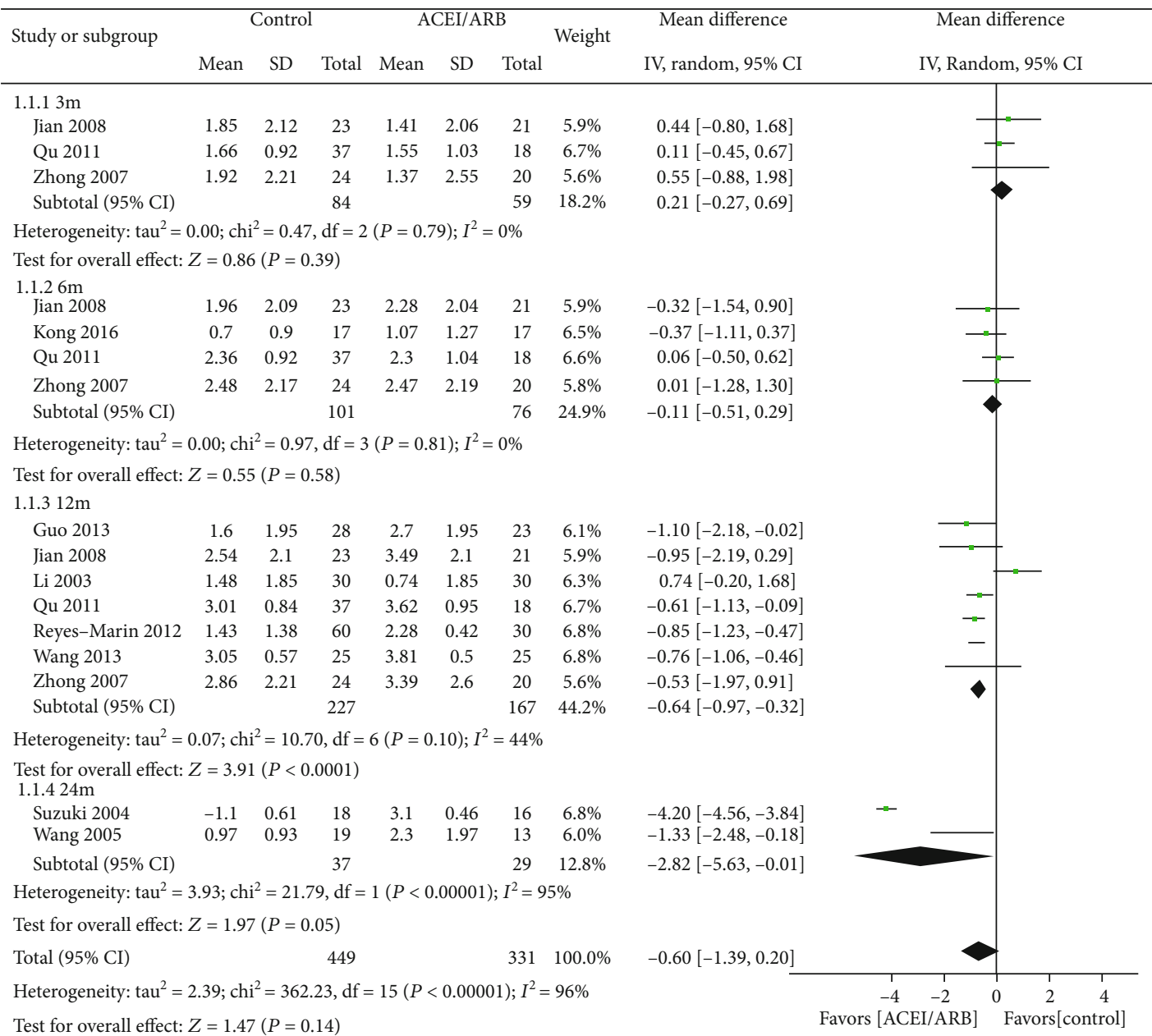

Test for subgroup differences: $\mathrm{chi}^{2}=12.61, \mathrm{df}=3(P=0.006) ; I^{2}=76.2 \%$

FIGURE 3: Change of residual GFR in the ACEI/ARB group versus placebo or other active agent group.

previous studies, our review included more RCTs focused on the residual renal function [36, 37]. The present study does have several limitations. First, the number and sample sizes of trials were too small. The observed different effect should be interpreted cautiously. Second, the majority of the included population were Asians, which might limit the gen- eralizability of the findings. In addition, heterogeneity of the outcome measurement and definitions and methodological quality of included studies were likely to profound the effects estimate of the findings. Therefore, high-quality RCTs with large sample size are needed to shed light on the impact of the present findings in PD patients. 


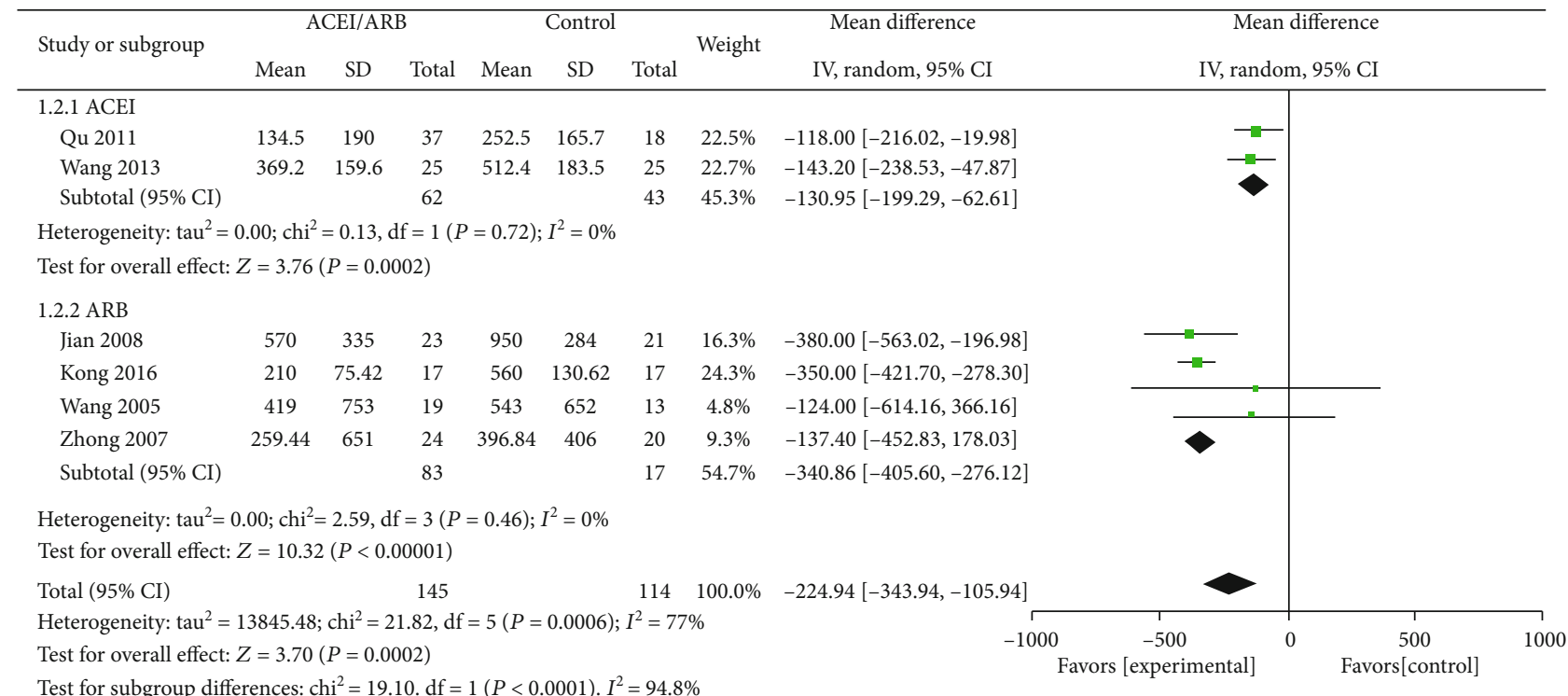

FIGURE 4: Change of urine volume in the ACEI/ARB group versus placebo or other active agent group.

\section{Conclusion}

In the present study, we found that use of ACEIs and ARBs, especially long-term treatment, decreased the decline of the residual renal function in patients on PD. ACEIs and ARBs do not cause an additional risk of side effects. Further studies are still needed to shed light on the impact of ACEIs and $\mathrm{ARBs}$ on the residual renal function in PD patients.

\section{Conflicts of Interest}

The authors declare that they have no conflict of interests.

\section{Authors' Contributions}

YY designed and conducted the research. LD and LZL searched the database. LD and JJY collected and analyzed the data. YY and LD wrote the draft; all authors read, reviewed, and approved the final manuscript.

\section{Supplementary Materials}

Additional file 1 Pubmed. Additional Figure 1: change of $\mathrm{RRF}$ in the ACEI/ARB group versus placebo or other active agent group at mo 12. Additional Figure 2: effect of ACEI or ARB compared with placebo or other active agents on anuria. Additional Figure 3: change of kt/v in the ACEI/ARB group versus placebo or other active agent group. Additional Figure 4: change of urinary protein excretion in the ACEI/ARB group versus placebo or other active agent group. Additional Figure 5: change of weekly creatinine clearance in the ACEI/ARB group versus placebo or other active agent group. (Supplementary Materials)

\section{References}

[1] J. I. Shen, A. B. Saxena, M. E. Montez-Rath, T. I. Chang, and W. C. Winkelmayer, "Angiotensin-converting enzyme inhibi- tor/angiotensin receptor blocker use and cardiovascular outcomes in patients initiating peritoneal dialysis," Nephrology Dialysis Transplantation, vol. 32, no. 5, pp. 862-869, 2017.

[2] F. F. Hou, X. Zhang, G. H. Zhang et al., "Efficacy and safety of benazepril for advanced chronic renal insufficiency," The New England journal of medicine, vol. 354, no. 2, pp. 131-140, 2006.

[3] S. Andersen, L. Tarnow, P. Rossing, B. V. Hansen, and H. H. Parving, "Renoprotective effects of angiotensin II receptor blockade in type 1 diabetic patients with diabetic nephropathy," Kidney international, vol. 57, no. 2, pp. 601-606, 2000.

[4] J. M. Bargman, K. E. Thorpe, D. N. Churchill, and CANUSA Peritoneal Dialysis Study Group, "Relative contribution of residual renal function and peritoneal clearance to adequacy of dialysis: a reanalysis of the CANUSA study," Journal of the American Society of Nephrology, vol. 12, no. 10, pp. 21582162, 2001.

[5] R. Paniagua, D. Amato, E. Vonesh et al., "Effects of increased peritoneal clearances on mortality rates in peritoneal dialysis: ADEMEX, a prospective, randomized, controlled trial," Journal of the American Society of Nephrology, vol. 13, no. 5, pp. 1307-1320, 2002.

[6] F. Termorshuizen, J. C. Korevaar, F. W. Dekker et al., "The relative importance of residual renal function compared with peritoneal clearance for patient survival and quality of life: an analysis of the Netherlands cooperative study on the adequacy of Dialysis (NECOSAD )-2," American journal of kidney diseases : the official journal of the National Kidney Foundation, vol. 41, no. 6, pp. 1293-1302, 2003.

[7] A. Y. M. Wang, M. M. M. Sea, R. Ip et al., "Independent effects of residual renal function and dialysis adequacy on actual dietary protein, calorie, and other nutrient intake in patients on continuous ambulatory peritoneal dialysis," Journal of the American Society of Nephrology, vol. 12, no. 11, pp. 24502457, 2001.

[8] M. K. Menon, D. M. Naimark, J. M. Bargman, S. I. Vas, and D. G. Oreopoulos, "Long-term blood pressure control in a cohort of peritoneal dialysis patients and its association with residual renal function," Nephrology Dialysis Transplantation, vol. 16, no. 11, pp. 2207-2213, 2001. 
[9] P. K. T. Li, K. M. Chow, T. Y. H. Wong, C. B. Leung, and C. C. Szeto, "Effects of an angiotensin-converting enzyme inhibitor on residual renal function in patients receiving peritoneal Dialysis," Annals of Internal Medicine, vol. 139, no. 2, pp. 105-112, 2003.

[10] H. Suzuki, Y. Kanno, S. Sugahara, H. Okada, and H. Nakamoto, "Effects of an angiotensin II receptor blocker, valsartan, on residual renal function in patients on CAPD," American journal of kidney diseases : the official journal of the National Kidney Foundation, vol. 43, no. 6, pp. 1056-1064, 2004.

[11] L. Zhang, X. Zeng, P. Fu, H. M. Wu, and Cochrane Kidney and Transplant Group, “Angiotensin-converting enzyme inhibitors and angiotensin receptor blockers for preserving residual kidney function in peritoneal dialysis patients," The Cochrane database of systematic reviews, vol. 6, 2014.

[12] A. Takahashi, H. Takase, T. Toriyama et al., "Candesartan, an angiotensin II type-1 receptor blocker, reduces cardiovascular events in patients on chronic haemodialysis-a randomized study," Nephrology, dialysis, transplantation, vol. 21, no. 9, pp. 2507-2512, 2006.

[13] H. Suzuki, Y. Kanno, S. Sugahara et al., "Effect of angiotensin receptor blockers on cardiovascular events in patients undergoing hemodialysis: an open-label randomized controlled trial," American journal of kidney diseases, vol. 52, no. 3, pp. 501-506, 2008.

[14] L. Guo and X. He, "Effects of valsartan on peritoneal function in patients undergoing peritoneal dialysis," International Journal of Transplantation and Hemopurification, vol. 11, no. 5, pp. 38-41, 2013.

[15] J. Guan, S. Gong, and X. Wu, "Influence of Astragalus combined with telmisartan on the insulin resistance of nondiabetic peritoneal dialysis patients," Chinese General Practice, vol. 18, no. 30, pp. 3717-3721, 2015.

[16] H. Jian, Z. Zhou, and H. Cui, "Effects of valsartan on residual renal function and microinflammation in peritoneal dialysis patients," Modern Medicine Journal of China, vol. 7, pp. 911,2008

[17] K. Xiangdong, S. Guomin, X. U. Jinhua, C. Xiaorong, Q. I. Ling, and S. Weihong, "Influence of irbesartan on microinflammation state and residual renal function in peritoneal dialysis patients China Modern Doctor," China Modern Doctor, vol. 54, no. 20, pp. 10-21, 2016.

[18] Q. U. Liaozhun, T. A. N. G. Xiaoling, and H. U. A. N. G. Dongjin, "Effect of benazepril on residual renal function in maintenance dialysis patients," HeBei Medicine Journal, vol. 17, no. 5, pp. 592-595, 2011.

[19] F. A. Reyes-Marín, C. Calzada, A. Ballesteros, and D. Amato, "Comparative study of enalapril vs. losartan on residual renal function preservation in automated peritoneal dialysis. A randomized controlled study," Revista de investigacion clinica, vol. 64, no. 4, pp. 315-321, 2012.

[20] A. Shigenaga, K. Tamura, T. Dejima et al., "Effects of angiotensin II type 1 receptor blocker on blood pressure variability and cardiovascular remodeling in hypertensive patients on chronic peritoneal dialysis," Nephron Clinical practice, vol. 112, no. 1, pp. c31-c40, 2009.

[21] H. Suzuki, H. Nakamoto, H. Okada, S. Sugahara, and Y. Kanno, "A selective angiotensin receptor antagonist, valsartan, produced regression of left ventricular hypertrophy associated with a reduction of arterial stiffness," Advances in peritoneal dialysis Conference on Peritoneal Dialysis, vol. 19, pp. 59-66, 2003.
[22] J. Wang and M. Xiao, "Protective effects of valsartan on residual renal function in patients on CAPD," Chinese Journal of Blood Purification, vol. 4, no. 11, pp. 605-606, 2005.

[23] X. Wang and W. Ren, "Effects of benazepril hydrochloride tablets on peritioneal function and residual renal function in peritoneal dialysis patients," Chinese Journal of Clinical Healthcare, vol. 16, no. 2, pp. 158-160, 2013.

[24] H. Zhong, C. H. Sha, T. L. Cui et al., "Effects of irbesartan on residual renal function in peritoneal dialysis patients," Chinese Journal of Nephrology, vol. 23, no. 7, pp. 413-416, 2007.

[25] C. H. Chen, J. Perl, and I. Teitelbaum, "Prescribing highquality peritoneal dialysis: the role of preserving residual kidney function," Peritoneal dialysis international : journal of the International Society for Peritoneal Dialysis, vol. 40, no. 3, pp. 274-281, 2020.

[26] M. Haag-Weber, "The impact of residual renal function on survival," Nephrology, dialysis, transplantation : official publication of the European Dialysis and Transplant Association European Renal Association., vol. 23, no. 7, pp. 2123-2126, 2008.

[27] J. I. Shen, A. B. Saxena, S. Vangala, S. K. Dhaliwal, and W. C. Winkelmayer, "Renin-angiotensin system blockers and residual kidney function loss in patients initiating peritoneal dialysis: an observational cohort study," BMC nephrology, vol. 18, no. 1, p. 196, 2017.

[28] I. Kolesnyk, M. Noordzij, F. W. Dekker, E. W. Boeschoten, and R. T. Krediet, "Treatment with angiotensin II inhibitors and residual renal function in peritoneal dialysis patients," Peritoneal dialysis international : journal of the International Society for Peritoneal Dialysis, vol. 31, no. 1, pp. 53-59, 2011.

[29] H. Hidaka and T. Nakao, "Preservation of residual renal function and factors affecting its decline in patients on peritoneal dialysis," Nephrology, vol. 8, no. 4, pp. 184-191, 2003.

[30] M. Haag-Weber, R. Kramer, R. Haake et al., "Low-GDP fluid (Gambrosol trio(R)) attenuates decline of residual renal function in PD patients: a prospective randomized study," Nephrology, dialysis, transplantation, vol. 25, no. 7, pp. 2288-2296, 2010.

[31] C. T. Liao, Y. M. Chen, C. C. Shiao et al., "Rate of decline of residual renal function is associated with all-cause mortality and technique failure in patients on long-term peritoneal dialysis," Nephrology, dialysis, transplantation : official publication of the European Dialysis and Transplant Association - European Renal Association, vol. 24, no. 9, pp. 2909-2914, 2009.

[32] J. F. Medcalf, K. P. Harris, and J. Walls, "Role of diuretics in the preservation of residual renal function in patients on continuous ambulatory peritoneal dialysis," Kidney International, vol. 59, no. 3, pp. 1128-1133, 2001.

[33] S. L. Lui, S. U. K. W. A. I. Cheng, F. Ng et al., "Cefazolin plus netilmicin versus cefazolin plus ceftazidime for treating CAPD peritonitis: effect on residual renal function," Kidney International, vol. 68, no. 5, pp. 2375-2380, 2005.

[34] U. C. Brewster, J. F. Setaro, and M. A. Perazella, "The reninangiotensin-aldosterone system: cardiorenal effects and implications for renal and cardiovascular disease states," The American journal of the Medical Sciences, vol. 326, no. 1, pp. 15-24, 2003.

[35] C. K. Wu, Y. H. Yang, J. M. J. Juang et al., "Effects of angiotensin converting enzyme inhibition or angiotensin receptor blockade in dialysis patients: a nationwide data survey and propensity analysis," Medicine, vol. 94, no. 3, p. e424, 2015. 
[36] Y. Liu, X. Ma, J. Zheng, J. Jia, and T. Yan, "Effects of angiotensin-converting enzyme inhibitors and angiotensin receptor blockers on cardiovascular events and residual renal function in dialysis patients: a meta-analysis of randomised controlled trials," BMC Nephrology, vol. 18, no. 1, p. 206, 2017.

[37] A. Akbari, G. Knoll, D. Ferguson, B. McCormick, A. Davis, and M. Biyani, "Angiotensin-converting enzyme inhibitors and angiotensin receptor blockers in peritoneal dialysis: systematic review and meta-analysis of randomized controlled trials," Peritoneal dialysis international : journal of the International Society for Peritoneal Dialysis, vol. 29, no. 5, pp. 554561, 2009. 